\title{
Failure Analysis of Welding Insulation Cylinder
}

\author{
Hua $\mathrm{Li}^{1, \mathrm{a}}$, Lian Wang ${ }^{1, \mathrm{~b}}$, Zhongming $\mathrm{Lu}{ }^{1, \mathrm{c}}$, Kexiu Liu ${ }^{1, \mathrm{~d}}$, Weijian $\mathrm{Qu}{ }^{1, \mathrm{e}}$, \\ Jinmei Lin ${ }^{1, f}$ \\ ${ }^{1}$ Guangzhou Special Pressure Equipment Inspection and Research Institute, \\ 510663 Guangzhou, China

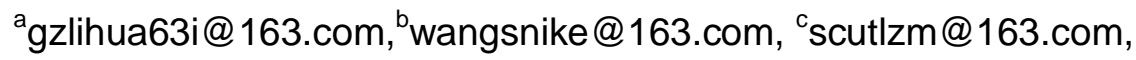 \\ d79666663@qq.com, ${ }^{e} 1536919184 @ q q . c o m,{ }^{f}$ gzspei@qq.com
}

Keywords: cylinder; welding; explosion; failure analysis

Abstract: In order to analyze the failure cause of welding insulation cylinder, to which explosion had happened, in a filling station, a series of experiments and analysis were taken, including macroscopic analysis, chemical composition analysis, fracture Analysis, metallographic examination and so on. The results showed that irrational structure led to fatigue cracking of fillet weld, connecting the inner-shell and neck pipe, and then pure oxygen continuously leaked into the vacuum interlayer. Friction heat or static electricity generating with the leaking finally caused combustion and explosion of flammable insulation materials in the interlayer. At the end, proposals were put forward.

\section{Introduction}

On May 20, 2015. A welding insulation cylinder, with full liquid oxygen, in the dangerous chemicals truck of a filling station exploded after being moved. Explosion occurred in the interior of the truck, with apparent red fire. The cylinder was used to fill LN2 (LO2, LAr), and made in September, 2008. The shell of it was made of OCr18Ni9, and the upper and lower heads were made of SUS304. To find out the cause of the explosion ${ }^{[1]}$, series of experiments and analysis were carried out.

\section{Macroscopic Analysis}

\section{Cylinder Morphology after the Accident}

After accident, macroscopic morphologies of the cylinder were as follows:

1) The outer-shell of the cylinder had serious cracking and deformation. There were obvious dark combustion products on its inner wall, with gray granular substances locally adhering to it. While the damage of the inner-shell was relatively slight. The two heads were both concave locally, and there were obvious dark combustion products on its outer wall.

2) Fillet welds, connecting the neck pipe, inner and outer shells, both cracked along the whole circle. In addition, the fillet weld, connecting the upper head of inner-shell and neck pipe, cracked along the weld toe.

3) Limited buttress plate of the inner-shell, which was welded on the inner surface of the outer-shell's lower head, bent and shed.

4) There was trace of repair on the vacuuming pipe of cylinder. The deformation of the nozzle 
was visible. In the inner wall of nozzle, there were obvious combustion residues, and no residual sealant or vacuum stopper.

5) No heat insulating materials remained in the vacuum interlayer.

In conclusion, the rupture of the outer-shell was due to chemical explosion in the vacuum interlayer, while the structure of the inner-shell was intact. Preliminary judgment was that oxygen leaked into the interlayer from the fillet weld, which connected the upper head of the inner-shell and reinforcing ring.

\section{Actual Structure of Cylinder}

The inner and outer shells of the welding insulation cylinder were connected by neck pipe through welding. The inner-shell obtained a single-ended support using the way of neck suspension. In movement and transportation, the inner-shell would shake. The impact force when filling liquid oxygen, could also cause the inner-shell to shake. Therefore, the neck pipe needed to bear the inertial dynamic load caused by the shaking of inner-shell, which led that fillet welds connecting the neck pipe and the inner and outer shells were the weakest parts of the cylinder.

Typically, there was supporting structure at the bottom of inner-shell to limit displacement, mainly to prevent the inner-shell laterally shaking during its use. Secondly, the supporting structure could also limit the sinking amplitude of the inner-shell, and then relieve stress concentration of fillet weld.

Through dissection of another cylinder, called comparative cylinder, and inspection of the cylinder's design drawings provided by the manufacturer, the actual structures of supporting structure and outer-shell's upper head did not exactly match the design drawings. The following problems were found out:

After the accident, the central portion of the outer-shell's lower head was convex, and there was clear trace of impact and damage caused by circular object. Limited buttress plate bent. Similar situations existed on the comparative cylinder, getting back from the accident scene. All above meant stiffness of the supporting structure was insufficient, which could not restrict the shaking of the inner-shell effectively and led to collapse of the outer-shell's upper head. Then there was serious stress concentration at the fillet weld connecting the neck pipe, the inner and outer shells.

There was a reinforcing ring to reinforce at the opening portion of the inner-shell's upper head, which connected the inner-shell's upper head and neck pipe through two fillet welds. But the thickness and diameter of the reinforcing ring were both small, resulting in two fillet welds with a close proximity, which affected the performance of materials and structures for dual heat in the process of welding.

\section{Measurement of Wall-Thickness}

Measuring the wall-thicknesses of heads and shell of the inner cylinder, and making comparison with the design thickness. As it is shown in the Tab.1. The minimum thicknesses measured were all slightly lower than the design thicknesses, which were in the range of negative deviation.

Tab.1 Comparison of actual and design thicknesses(mm)

\begin{tabular}{cccc}
\hline & Upper head & Lower head & Shell \\
\hline Minimum thickness & 2.12 & 2.11 & 2.34 \\
Design thickness & 2.16 & 2.16 & 2.40 \\
\hline
\end{tabular}




\section{Chemical Composition Analysis}

The sample, taken from the base metal of inner-shell's upper head, was used for spectral analysis. Known from the experimental data in Tab.2, the chemical composition of material met the standards ${ }^{[2]}$.

Tab.2 Data of chemical composition (Mass fraction, \%)

\begin{tabular}{cccccccc}
\hline No. & C & Si & Mn & P & S & Cr & Ni \\
\hline 1 & 0.04 & 0.37 & 1.11 & 0.033 & 0.0065 & 18.54 & 8.01 \\
2 & 0.04 & 0.37 & 1.11 & 0.032 & 0.0068 & 18.50 & 8.02 \\
3 & 0.04 & 0.38 & 1.12 & 0.031 & 0.0063 & 18.43 & 7.98 \\
SUS304/JIS G3459 & $\leqslant 0.08$ & $\leqslant 1.00$ & $\leqslant 2.00$ & $\leqslant 0.040$ & $\leqslant 0.030$ & $18.00^{\sim} 20.00$ & $8.00^{\sim} 11.00$ \\
\hline
\end{tabular}

\section{Fracture Analysis}

\section{Macroscopical Morphology Observation}

The fillet weld between the inner-shell's upper head and reinforcing ring fractured along the whole circle weld toe. As shown in Fig.1. Fig.2 showed fracture was flush and there were two obvious tearing rips belonging to the final fracture zone of the weld.

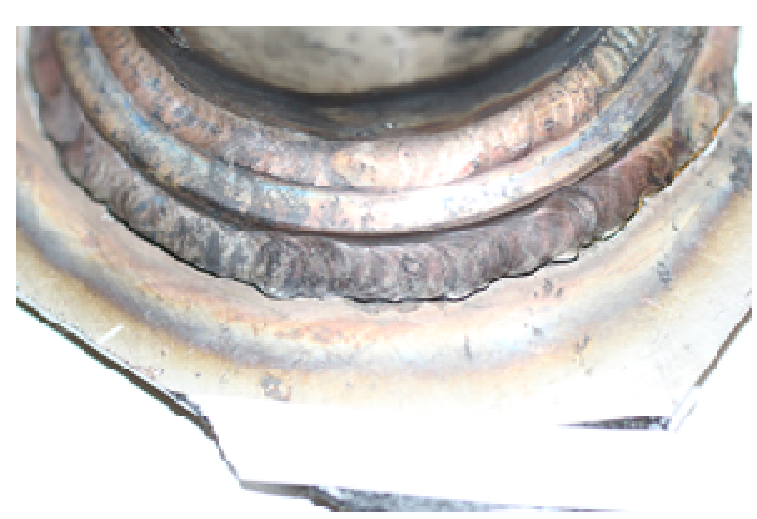

Fig.1 Fillet weld fracture

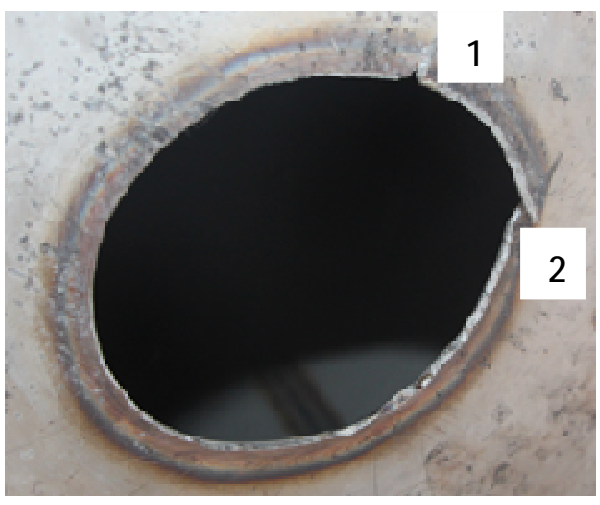

Fig.2 Tearing rips(1-Tearing rip, 2-Tearing rip)

\section{Morphology Analysis}

As it is shown in Fg.3, samples from the weld of fracture between the inner-shell's upper head and the reinforcing ring were used for morphology analysis. The result showed fatigue fracture characteristics with multiple fatigue sources. As shown in Fig.4, there were fatigue striations existing on the whole circle fracture and fatigue steps existing on the local area. In addition, the fatigue striations on the fracture between the position " $\mathrm{M}$ " and the position " $\mathrm{N}$ " covered almost the entire cross-section, which meant crack and leak originated in this region ${ }^{[3]}$. 


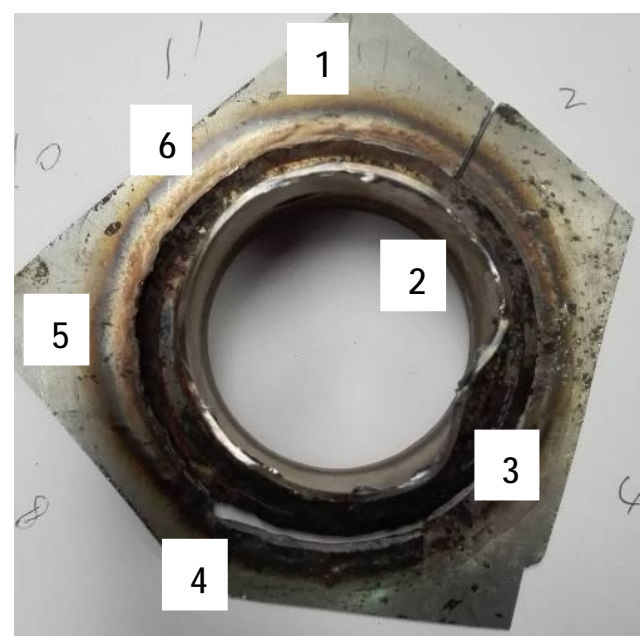

Fig.3 Diagram of fracture morphology (1- Inner-shell's

Upper head, 2-Neck pipe, 3-Reinforcing ring,

4-Fracture, 5-Position "M", 6-Position "N")

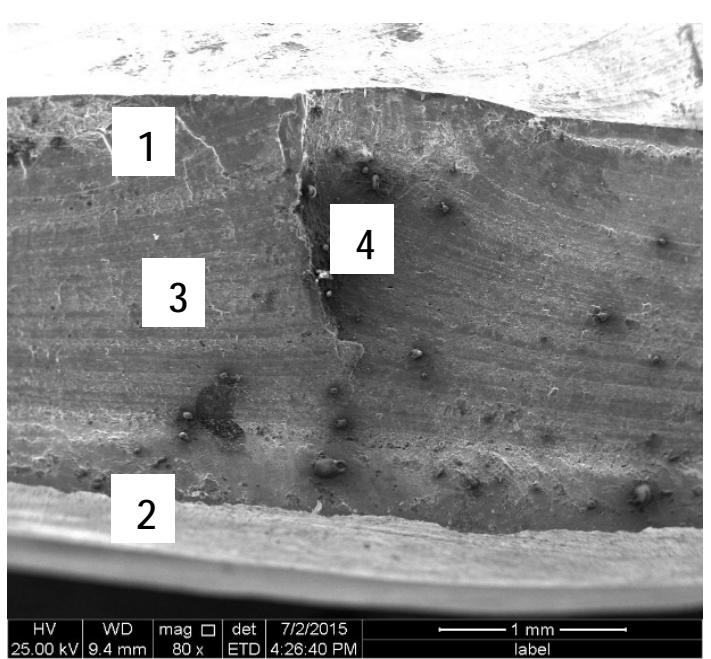

Fig.4 Fracture morphology (Reinforcing ring side, 11th)

(1-Outer wall, 2-Inner wall, 3- Fatigue striations, 4

-Fatigue steps )

\section{Metallographic Examination}

Sampling from the fillet weld, connecting the inner-shell's upper head and neck pipe, and its adjacent areas for metallographic examinations ${ }^{[4]}$, shown in Fig.5. The results were as follows:

(1)The microstructure of base metal in the inner-shell's upper head, reinforcing ring, and neck pipe, was twinning austenite (in Fig.6 Fig.8), without exceptions. While in weld, was austenite of columnar crystals, and there was apparent carbide precipitation in base metal of heat affected zone (in Fig.9 Fig.10).

(2)Fracture was located on the heat affected zone of fillet weld, connecting the inner-shell's upper head and reinforcing ring, near the side of head (in Fig.9 Fig.11), and incomplete fusion existed in the fillet weld (in Fig.12).

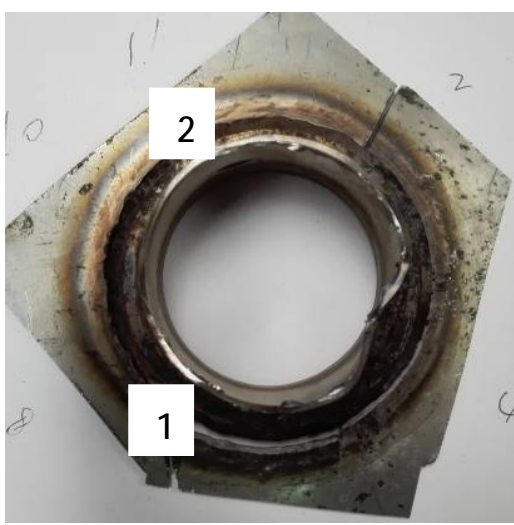

Fig.5 Diagram of sampling (1-Position “A”, 2-Position "B”)

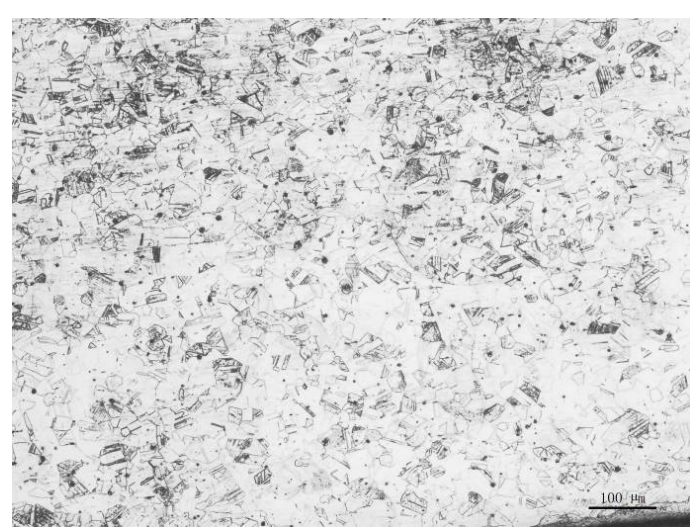

Fig.6 Base metal of upper header (100×, Position "B”) 


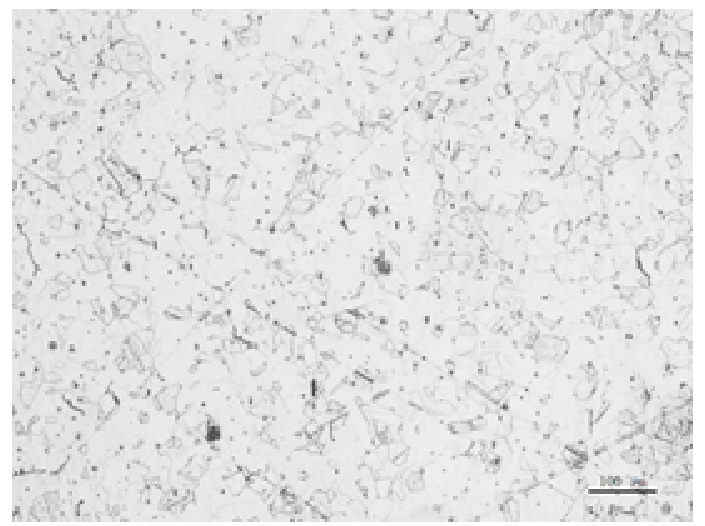

Fig.7 Base metal of reinforcing ring $(100 \times$, Position " $A$ ")

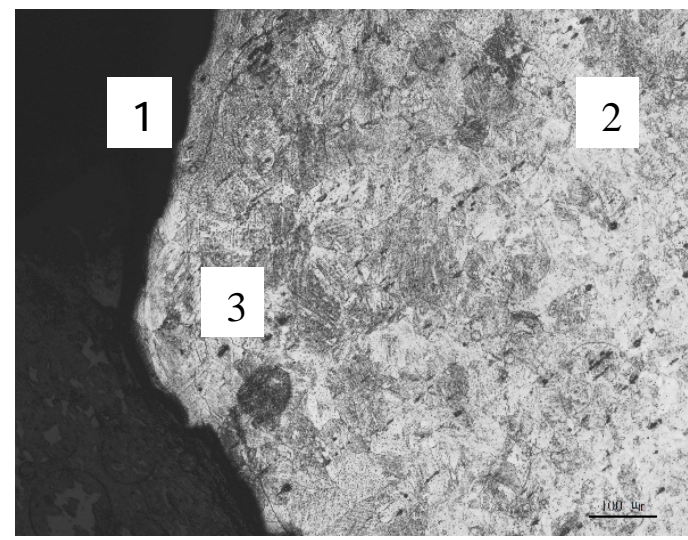

Fig.9 Fracture of inner-shell's upper head (100×, Position "B") (1- Fracture, 2- Base metal, 3- Heat affected zone of inner-shell's upper head)

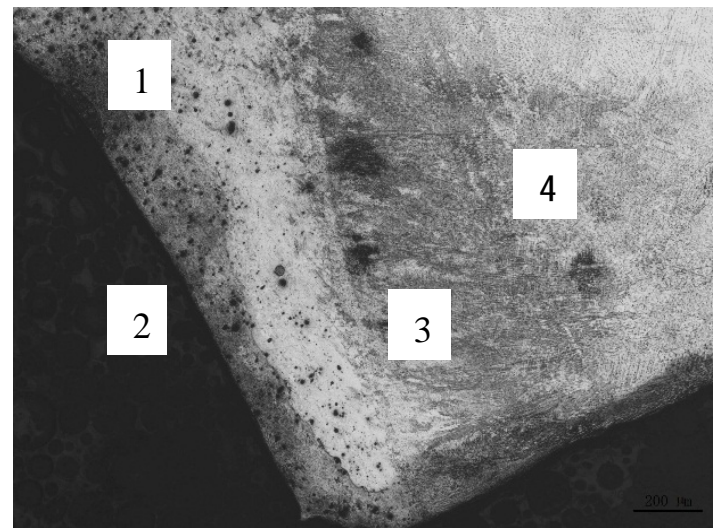

Fig.11 Fracture of reinforcing ring (50×, Position "A")

(1- Heat affected zone of inner-shell's upper heat, 2Fracture, 3- Fusion line, 4-Weld)

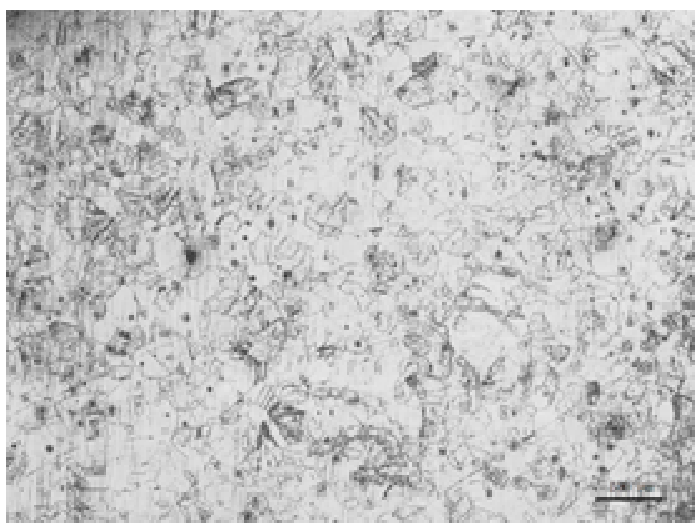

Fig.8 Base metal of neck pipe (100×, Position "A")

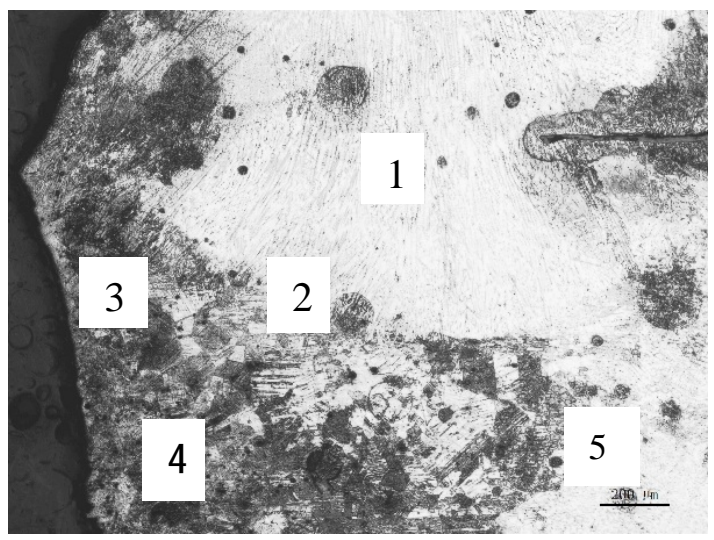

Fig.10 Fracture of reinforcing ring (50×, Position "A") (1- The outside of weld, 2-Fusion line, 3- Fracture,4Heat affected zone of inner-shell's upper head, 5- The inside of weld )

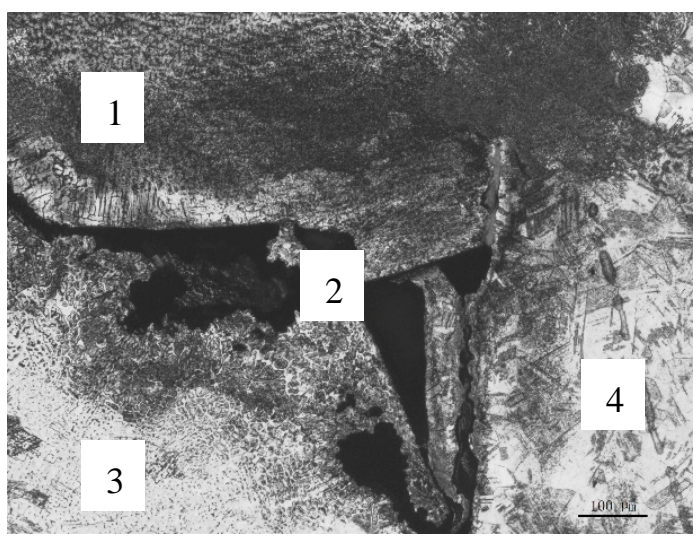

Fig.12 Fillet weld (100×, Position “A”)

(1- The outside of weld, 2- Incomplete fusion area,3-

The inside of weld, 4- Reinforcing ring)

\section{Combustion Performance of Insulation Materials}

Four different samples were taken from the interlayer of comparative cylinder, used for tests of combustion performance. Insulation materials were wound on the outer wall of the inner-shell, and from the inside to the outside were yellowish and fibrous insulation material (1\#), aluminum foil 
(3\#), white and fibrous material (4\#), and white paper-like material (2\#), with the aluminum foil at the outermost layer. According to the oxygen index and ignition point, in Tab.3, shown that, the 2th sample was flame retardant material, while the others were combustible, and ignition points of all the four were higher than $500^{\circ} \mathrm{C}$.

Tab.3 Data of combustion performance

\begin{tabular}{ccccc}
\hline Number & $1 \#$ & $2 \#$ & $3 \#$ & $4 \#$ \\
\hline Oxygen index $(\%)$ & 18.55 & 40.0 & 18.75 & 18.15 \\
Ignition point $\left({ }^{\circ} \mathrm{C}\right)$ & 570 & $>1000$ & 880 & 565 \\
Combustion performance levels & B3 & B1 fire & B3 & B3 \\
GB8624-2012 & combustible & nonflammable & combustible & combustible \\
\hline
\end{tabular}

Dark attachments, which were taken from the inner wall of outer-shell, were used for X-ray diffraction analysis of chemical composition. Results showed that the main components of attachments were $\mathrm{Al}, \mathrm{CaO}$ and $\mathrm{SiO} 2$ (in Fig.13), which should be the combustion products of insulation materials (aluminum foil, mineral fibers, etc.)

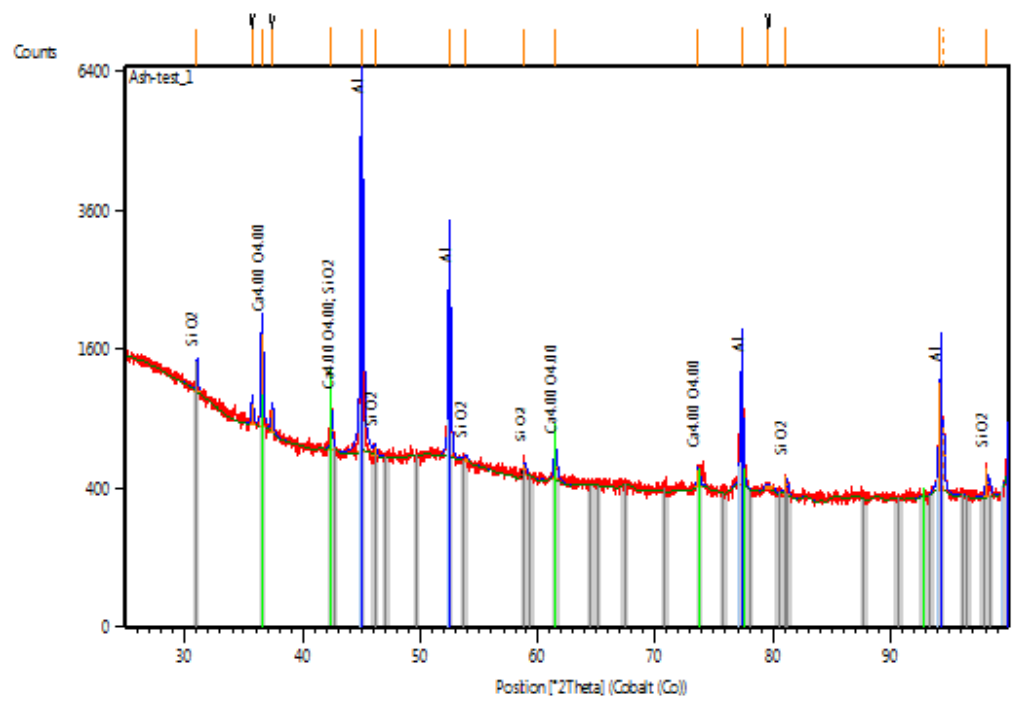

Fig.13 Spectrum of chemical composition

\section{Cause Analysis}

(1) Irrational structure caused the fillet weld occurring fatigue cracking prematurely.

The design drawings provided by the manufacturer showed the actual limited buffer structure did not exactly match the design, which could not restrict the shaking and vibration of the inner-shell caused by moving and filling and resulted in fillet weld occurring fatigue cracking prematurely for stress concentration. The result of fracture analysis showed that crack originated from the outside weld toe of fillet connecting the inner-shell's upper head and the reinforcing ring, and fracture had obvious fatigue expansion characteristics.

(2) There was combustion process in the vacuum interlayer of the cylinder before explosion.

The result showed most of insulation materials in the interlayer of comparative cylinder were flammable materials, which provided fuel for combustion. Friction heat or static electricity generating with the media leaking after the rupture of outer-shell' fillet weld, provided ignition energy for combustion. Finally, in the environment with the pure oxygen leaking, the insulating materials were ignited and burnt fiercely, and then led to the explosion. In addition, there were red flames with the instant explosion, and there was dark combustion product on the inside of 
inner-shell. All above showed that there was combustion process in the vacuum interlayer of cylinder before explosion.

(3) Safety hazards existed for the vacuum maintenance of cylinder.

There was trace of welding, repair and replacement on the vacuuming pipe of accidental cylinder. The similar phenomenon was found on the other welding insulation cylinders in the accident scene. And the situation that the yellow grease was used of seal existed, which had safety hazards as follows. When oxygen releasing into the interlayer discharged from vacuum interface at a high speed, and exposed to yellow grease, the combustion and explosion could happen. When oil diffusion pump was used for evacuated maintenance, the oil vapor in the pump might suck into the interlayer of cylinder and adsorb on the insulating material. Combustion and explosion might also take place, once oxygen leaked into the interlayer.

\section{Conclusion and Suggestion}

Irrational structure led to the fatigue cracking of fillet weld, connecting the inner-shell and neck pipe, and then pure oxygen continuously leaked into the vacuum interlayer. Friction heat or static electricity generating with the leaking finally caused combustion and explosion of flammable insulation materials in the interlayer. Proposals were made from the following aspects.

(1)Further expand the spot check of welding insulation cylinders, made in the same manufacturer, and with the same model and batch, and determine whether there are the similar problems and systemic risks.

(2)Strengthen regular inspection of welding insulation cylinders, and overdue cylinders without inspection are prohibited to filling and using.

(3)Strengthen maintenance and management for the welding insulation cylinders in-service, and make sure the maintenance is carried out by the qualified institutions.

\section{Reference}

[1] Jincheng Niu, Failure analysis of industrial gas cylinders, J. Industrial Technology, Lanzhou, 2008, Vol.37(4):p.32.

[2] Zongren Gao, Handbook of world stainless steel and heat-resistant steel, J. Shanxi Science and Technology Press, Taiyuan, 2006, p.14 19.

[3] Zhengyi Liu, Liansheng $\mathrm{Wu}$, Atlas of equipment failure analysis. Guangdong Science and Technology Press, Guangzhou, 1990, p.272 273.

[4] Songzan Ren, Jingjiang Zhang, Zhiru Chen, Steel microstructure map. Shanghai Science and Technology Literature Publishing House, Shanghai, 2003. 